\title{
A Novel Approach to Noncommutativity in Planar Quantum Mechanics
}

\author{
Rabin Banerje日], \\ S. N. Bose National Centre for Basic Sciences \\ JD Block, Sector III, Salt Lake City, Calcutta -700 098, India.
}

\begin{abstract}
Noncommutative algebra in planar quantum mechanics is shown to follow from 't Hooft's recent analysis on dissipation and quantization. The noncommutativity in the coordinates or in the momenta of a charged particle in a magnetic field with an oscillator potential are shown as dual descriptions of the same phenomenon. Finally, noncommutativity in a fluid dynamical model, analogous to the lowest Landau level problem, is discussed.
\end{abstract}

\section{Introduction}

Noncommutative spaces arise as brane configurations in string theory as well as in the matrix model of M-theory [四]. The appearance of this noncommutativity is quite similar to a corresponding effect in the Landau problem of a charged particle moving on a plane, subjected to a strong perpendicular magnetic field. It is therefore desirable to investigate the noncommutative effects in planar quantum mechanics to get a deeper understanding of this phenomenon in the context of string or matrix models. Some studies [2] along this direction have been performed. But these are done in a way that either requires the occurence of noncommutativity (in the momenta) due to the presence of a magnetic field, or just as a postulate (noncommutativity in the coordinates).

In this paper we would like to address the question of how and why such noncommutative structures appear in planar quantum mechanics and provide a systematic way of obtaining them. For this we adopt a recent approach advocated by 't Hooft [3, [1] to study quantum mechanics. In this approach the classical equations are written in a formulation using quantum mechanical notation. However the quantum mechanical hamiltonian does not have a lower bound, which is the price to be paid for expressing a classical system quantum mechanically. Constarints, motivated by information loss or dissipation, are now imposed leading to a well defined bounded hamiltonian as well as to an apparent quantisation of the orbits.

The point we make is that a modification of the hamiltonian brought about by dissipation leads to a deformation in the symplectic structure. The original Poisson brackets are changed to

\footnotetext{
${ }^{1}$ rabin@bose.res.in
} 
a new set of brackets. This is worked out in details for the motion of a charged particle in a plane subjected to a magnetic field(Landau problem) and a quadratic potential. It naturally leads to a noncommutative algebra which is otherwise postulated. Thus, apart from illuminating the various noncommutative structures, the present work also places 't Hooft's observations in a different setting.

In section 2, we review the emergence of noncommutative coordinates for the motion of particles in the lowest Landau level. A strong magnetic field is necessary to effect the projection to this level. However, we show that it is possible to map the general Landau problem to a chiral oscillator model which is described by noncommuting variables. Thus the Landau problem for any magnetic field exhibits noncommutativity, albeit in a different set of coordinates. Indeed the mapping of the Landau problem to the chiral oscillator has also been used in the subsequent analysis.

The noncommutativity in the general problem of a charged particle in magnetic and electric fields, mentioned earlier, is discussed in section 3. This problem has recently generated considerable interest in both theoretical studies [2] and phenomenological applications [5]. The second order equation of motion is expressed as a pair of first order equations. In this version, 't Hooft's analysis, a synopsis of which is given below, is applicable. Noncommutativity in the coordinates or in the momenta are shown to be dual descriptions, corresponding to distinct polarisations chosen for converting a second order system to a first order system. The issue is further clarified by interpreting the results as different superpositions of the noncommutativity in the chiral oscillator models of section 2.

In section 4, a magnetohydrodynamical(MHD) field theory will be investigated from 't Hooft's formalism. A complete mapping with the lowest Landau level problem will be done, providing an alternative derivation of noncommutative MHD [6].

We present some concluding remarks in section 5 .

We end this section by briefly mentioning 't Hooft's analysis. This is basically a hamiltonian formulation where first order equations $\dot{q}_{i}=\left\{q_{i}, H\right\}=f_{i}(q)$, decoupled from the conjugate momenta $p_{i}$, are considered. The corresponding hamiltonian $H=\sum_{i} p_{i} f_{i}(q)$ obviously does not have a lower bound. This is cured by expressing $H$ as,

$$
H=H_{+}-H_{-}
$$

where

$$
H_{ \pm}=\frac{1}{4 \rho}(\rho \pm H)^{2}
$$

and $\rho$ is a positive definite function of $q_{i}$ satisfying

$$
\{\rho, H\}=0
$$

so that ordering ambiguities (upon quantization) in (2) are avoided. To get a lower bound for $H$, one imposes the constraint

$$
H_{-} \mid \psi>=0
$$


on the physical space. The constraint can be motivated by dissipation or information loss [3] . In this paper however we shall not be concerned with studying dissipative effects, but rather dwell on a completely new manifestation that has not been discussed either in the original papers [3] or in the subsequent applications [4]. The point is that a change from the original (unbounded) hamiltonian $H$ to the bounded positive (semi) definite hamiltonian $\rho$ leads to a modified algebra that can be obtained as follows,

$$
\dot{q}_{i}=\left\{q_{i}, \rho\right\}=\left\{q_{i}, q_{j}\right\} \partial_{j} \rho(q)
$$

To reproduce the original set of equations of motion, obviously $q_{i}$ can no longer be taken as commuting and must be calculated from,

$$
\left\{q_{i}, q_{j}\right\} \partial_{j} \rho(q)=f_{i}(q)
$$

leading to explicit noncommutative structures for the algebra of $q_{i}$. Furthermore if the brackets $\Lambda_{i j}=\left\{q_{i}, q_{j}\right\}$ are nondegenerate, the corresponding lagrangian can also be evaluated,

$$
L=\frac{1}{2} q_{i} \Lambda^{i j} \dot{q}_{j}-\rho(q)
$$

where $\Lambda^{i j}$ is the inverse of $\Lambda_{i j}$,

$$
\Lambda^{i j} \Lambda_{j k}=\delta_{k}^{i}
$$

\section{The Landau problem revisited}

The classical equations of motion for an electron of charge $-e$ moving in the $x_{1}-x_{2}$ plane under the influence of a constant perpendicular magnetic field $B$ are,

$$
m \ddot{x}_{i}=-\frac{e}{c} B \epsilon_{i j} \dot{x}_{j}
$$

whose general solution in the complex notation $Z=x_{1}+i x_{2}$, corresponds to motion in a circle of radius $R$,

$$
Z=R e^{i\left(\omega_{c} t+\delta\right)}
$$

Here $\delta$ is an arbitrary phase and the cyclotron frequency is,

$$
\omega_{c}=\frac{e B}{m c}
$$

The radius of the orbit is related to the tangential velocity $v$ by $v=\omega_{c} R$. The centre of the orbit is chosen at the origin, but it could be displaced to any other position $C(Z \rightarrow Z+C)$ without changing the physics.

The above equations of motion follow from the Lagrangian,

$$
L=\frac{m}{2} \dot{x}_{i}^{2}-\frac{e}{2 c} B \epsilon_{i j} x_{i} \dot{x}_{j}
$$


The canonical momentum is given by,

$$
p_{i}=\frac{\partial L}{\partial \dot{x}_{i}}=m \dot{x}_{i}+\frac{e}{2 c} B \epsilon_{i j} x_{j}
$$

while the hamiltonian is

$$
H=\frac{\pi_{i}^{2}}{2 m}=\frac{1}{2 m}\left(p_{i}-\frac{e}{2 c} B \epsilon_{i j} x_{j}\right)^{2}
$$

In analogy with the classical considerations it is possible to define a quantum orbit centre operator,

$$
C=Z+\frac{i \pi}{m \omega_{c}} ; C_{i}=x_{i}+\frac{i \pi_{i}}{m \omega_{c}}
$$

which is the source of the degeneracy in the Landau problem and satisfies the algebra,

$$
\left[C_{i}, C_{j}\right]=i \frac{\hbar c}{e B} \epsilon_{i j}
$$

Let us now specialise to the lowest Landau level problem by enforcing a strong magnetic field. Heuristically, the classical analysis shows that the particles are projected to their respective orbit centres $(Z=C)$, where they are frozen. The kinetic energy is completely quenched, but the algebra among the co-coordinates, given by (16), is noncommutative. The above result can be understood by naively setting $m=0$ in (12) (quenching of the kinetic term) in which case the lagrangian is first order leading to the fundamental brackets,

$$
\left[x_{i}, x_{j}\right]=i \frac{\hbar c}{e B} \epsilon_{i j}
$$

reproducing (16).

A more rigorous edge to these arguments is provided by using the projection technique developed in [7]. The basis of states in the lowest Landau level may be chosen as,

$$
\psi_{n}\left(Z, Z^{*}\right)=\frac{Z^{n}}{\sqrt{\left(2 l^{2}\right)^{n} n ! \pi}} \exp \left(-\frac{Z Z^{*}}{4 l^{2}}\right)
$$

where the magnetic length $l$ is defined by,

$$
l^{2}=\frac{\hbar c}{e B}
$$

The exponential factor, which is common to all eigenfunctions, is removed by formally defining a Hilbert space of analytic functions [7]. One now works with the polynomial factor only which, in contrast to the exponential factor, is analytic. In this scenario the projection of $Z$ is trivial $(Z \rightarrow Z)$ since it is already contained in the lowest Landau level. The projection of $Z^{*}$, on the other hand, leads to a differential operator [7] $Z^{*} \rightarrow-2 l^{2} \frac{\partial}{\partial Z}$. The fundamental algebra, after this projection, thus yields,

$$
\left[x_{1}, x_{2}\right]=\frac{1}{2 i}\left[Z^{*}, Z\right] \rightarrow \frac{1}{2 i}\left[-2 l^{2} \frac{\partial}{\partial Z}, Z\right]=i l^{2}
$$


showing the noncommutativity (17) among the coordinates.

Having reviewed the noncommutativity in the lowest Landau level, let us provide a mapping of the original Landau problem which manifests this property for any magnetic field. Classically or quantum mechanically, the Landau problem reduces to the motion of a one dimensional harmonic oscillator. Actually since the direction (clockwise or anticlockwise) of cyclotron motion of the charged particle is governed by the direction of the magnetic field(pointing up or down) one should regard it as the chiral form of the harmonic oscillator. The Lagrangian of a chiral oscillator is given by,

$$
L_{+}=\frac{1}{2} \epsilon_{i j} y_{i} \dot{y}_{j}-\frac{\omega}{2} y_{i}^{2}
$$

Eliminating any of the $y_{i}$ 's in favor of the other yields a 1-d harmonic oscillator in that variable with frequency $\omega$. The equation of motion is,

$$
\dot{y}_{i}=-\omega \epsilon_{i j} y_{j}
$$

It is easy to read-off the brackets and the hamiltonian directly from (21)

$$
\begin{gathered}
\left\{y_{i}, y_{j}\right\}=-\epsilon_{i j} \\
H=\frac{\omega}{2} y_{i}^{2}
\end{gathered}
$$

leading immediately to (22) using the hamilton equations.

The chirality comes from the sign of the kinetic term in (21). Computing the angular momentum,

$$
J=\epsilon_{i j} y_{i} p_{j}=\frac{H}{\omega}
$$

where $p_{i}=\frac{\partial L}{\partial \dot{y}_{i}}$ is the conjugate momentum. If the sign of the kinetic term is flipped so that

$$
L_{-}=-\frac{1}{2} \epsilon_{i j} y_{i} \dot{y}_{j}-\frac{\omega}{2} y_{i}^{2}
$$

we obtain $J=-\frac{H}{\omega}$. Note that $L_{-}$also reduces to a 1 -d harmonic oscillator by the variable elimination process. Thus $L_{ \pm}$characterize a pair of chiral oscillators rotating in an anticlockwise or clockwise direction. A suitable combination of such oscillators just leads to a two dimensional oscillator and has been used in the study of Zeeman effect and duality symmetry [8].

The Landau problem is now mapped to the chiral oscillator by using the correspondence,

$$
\sqrt{m} \epsilon_{i j} \dot{x}_{j} \rightarrow \sqrt{\omega} y_{i}
$$

where $\omega$ is identified with the cyclotron frequency $\omega_{c}$. As a check we find that the Landau hamiltonian maps to the chiral oscillator hamiltonian,

$$
\frac{m}{2} \dot{x}_{i}^{2} \rightarrow \frac{1}{2} \omega y_{i}^{2}
$$


The equation of motion(9) goes over to (22) while the noncommutative algebra of $\pi_{i}$ in (14) reproduces (23). Thus there is a complete mapping between the Landau model and the chiral oscillator. The coordinates in the latter satisfy a noncommutative algebra. The result is true for any value of the magnetic field $B$. The limit of the strong magnetic field, as necessary in usual treatments of noncommutativity in the lowest Landau level problem, is now irrelevant in this new set of variables.

It may be mentioned that the above mapping also follows by recalling the equivalence [9] between the self dual model,

$$
\mathcal{L}_{S D}=\frac{1}{2} f_{\mu} f^{\mu}-\frac{1}{2 m} \epsilon_{\mu \nu \lambda} f^{\mu} \partial^{\nu} f^{\lambda}
$$

and the Maxwell-Chern-Simons theory,

$$
\mathcal{L}_{M C S}=-\frac{1}{4} F_{\mu \nu} F^{\mu \nu}+\frac{m}{2} \epsilon_{\mu \nu \lambda} A^{\mu} \partial^{\nu} A^{\lambda}
$$

where the basic field $f_{\mu}$ gets identified with the dual field tensor $F_{\mu}$,

$$
f_{\mu}=F_{\mu}=\epsilon_{\mu \nu \lambda} \partial^{\nu} A^{\lambda}
$$

If a projection from the field theory models to the planar quantum mechanical models is done by ignoring the spatial derivatives and setting the time components $f_{0}, A_{0}$ to zero, the lagrangians (29) and (30) correspond respectively to the chiral oscillator and the Landau model. Then the map (31) is the analogue of (27). Other possibilities and consequences of such a projection have been discussed in [10].

This might be an appropriate place of explicitly working with the 't Hooft mechanism to realize the noncommutativity in the chiral oscillator, because equation (22) only involves the coordinates. The corresponding hamiltonian is,

$$
H=-\left(\omega \epsilon_{i j} y_{j}\right) p_{i}
$$

which yields (22) upon using $\dot{y}_{i}=\left\{y_{i}, H\right\}$. This hamiltonian is not bounded from below. Following 't Hooft's prescription discussed earlier, the physical hamiltonian $\rho$ is constructed so that it commutes with $H$. This is given by

$$
\rho=\frac{\omega}{2} y_{i}^{2} ; \quad\{\rho, H\}=0
$$

To reproduce (22) the symplectic structure gets modified to (23) so that

$$
\dot{y}_{i}=\left\{y_{i}, \rho\right\}=-\omega \epsilon_{i j} y_{j}
$$

The noncommutative bracket (23) is thereby reproduced, together with the hamiltonian (24). 


\section{Noncommutativity in the Landau problem with an os- cillator potential}

The equation of motion for a charged particle moving in the $x_{1}-x_{2}$ plane under the influence of a magnetic field $B$ with an oscillator potential is given by.]

$$
m \ddot{x}_{i}-B \epsilon_{i j} \dot{x}_{j}+k x_{i}=0
$$

Recall that the harmonic oscillator was recast in the form of a chiral oscillator by doubling the degrees of freedom which manifested noncommutative properties. Thus the first step is to rewrite the second order system into a pair of first order equations by doubling the degrees of freedom. There is a certain amount of flexibility in doing this, but for the moment we confine to the following choice.

$$
\dot{x}_{i}=\frac{1}{m} q_{i} ; \quad \dot{q}_{i}=B \epsilon_{i j} \dot{x}_{j}-k x_{i}
$$

Also $q_{i}$ satisfies an equation identical to (35),

$$
m \ddot{q}_{i}-B \epsilon_{i j} \dot{q}_{j}+k q_{i}=0
$$

The hamiltonian that yields the above set of first order equations is,

$$
H=\frac{1}{m} q_{i} \pi_{i}^{x}+\left(\frac{B}{m} \epsilon_{i j} q_{j}-k x_{i}\right) \pi_{i}^{q}
$$

where $\left(x_{i}, \pi_{i}^{x}\right)$ and $\left(q_{i}, \pi_{i}^{q}\right)$ are canonical pairs. This hamiltonian is not bounded from below. Following 't Hooft, a bounded hamiltonian follows upon dissipation. Explicitly this is found by constructing a positive definite $\rho$ that commutes with $H$ :

$$
\rho=\frac{1}{2}\left(\frac{q_{i}^{2}}{m}+k x_{i}^{2}\right) ; \quad\{\rho, H\}=0
$$

In order to generate the equations (36) from $\rho\left(\dot{x}_{i}=\left\{x_{i}, \rho\right\}, \dot{q}_{i}=\left\{q_{i}, \rho\right\}\right)$, it is obvious that the basic brackets are now given by,

$$
\left\{x_{i}, q_{j}\right\}=\delta_{i j}, \quad\left\{x_{i}, x_{j}\right\}=0, \quad\left\{q_{i}, q_{j}\right\}=B \epsilon_{i j}
$$

thereby revealing a noncommutative algebra. To make contact with known results, one interprets the hamiltonian $\rho$ in (39) as the usual harmonic oscillator hamiltonian with noncommutative momenta $\left(q_{i}\right)$ manifesting the presence of the magnetic field. It is straightforward to obtain the lagrangian since the matrix,

$$
\Lambda_{i j}=\left[\left\{\Gamma_{i}, \Gamma_{j}\right\}\right]=\left(\begin{array}{cc}
0 & \delta_{i j} \\
-\delta_{i j} & B \epsilon_{i j}
\end{array}\right)
$$

\footnotetext{
${ }^{2}$ We have rationalised $e=c=1$.
} 
of the coordinates $\Gamma=(x, q)$ possesses an inverse,

$$
\Lambda^{i j}=\left(\begin{array}{cc}
B \epsilon_{i j} & -\delta_{i j} \\
\delta_{i j} & 0
\end{array}\right)
$$

The lagrangian is given by,

$$
L=\frac{1}{2} \Gamma_{i} \Lambda^{i j} \dot{\Gamma}_{j}-\rho(\Gamma)=q_{i} \dot{x}_{i}+\frac{B}{2} \epsilon_{i j} x_{i} \dot{x}_{j}-\frac{1}{2}\left(\frac{q_{i}^{2}}{m}+k x_{i}^{2}\right)
$$

Explicitly the equations of motion from the lagrangian reproduce (36). Since $q_{i}$ is an auxiliary variable it can be eliminated by solving for it to yield,

$$
L=\frac{m}{2} \dot{x}_{i}^{2}+\frac{B}{2} \epsilon_{i j} x_{i} \dot{x}_{j}-\frac{1}{2} k x_{i}^{2}
$$

which is the standard lagrangian leading to (35).

Next, we show how noncommutativity in the coordinates, instead of the momenta, arise. As remarked before there is a freedom in splitting (35) into a dual set of first order equations. Consider the following polarisation,

$$
\dot{q}_{i}=-k x_{i}, \quad \dot{x}_{i}=\frac{q_{i}}{m}+\frac{B}{m} \epsilon_{i j} x_{j}
$$

This set also yields the equation (35) as well as (37). The desired hamiltonian is, therefore, given by,

$$
H=\left(-k x_{i}\right) \pi_{i}^{q}+\left(\frac{q_{i}}{m}+\frac{B}{m} \epsilon_{i j} x_{j}\right) \pi_{i}^{x}
$$

The physical hamiltonian $\rho$ is once again given by (39). However since the basic equations (45) are different from (36) the algebra (40) also gets modified to,

$$
\left\{x_{i}, q_{j}\right\}=\delta_{i j},\left\{q_{i}, q_{j}\right\}=0,\left\{x_{i}, x_{j}\right\}=\theta \epsilon_{i j}
$$

where the noncommutativity parameter $\theta=\frac{B}{\mathrm{~km}}$. Comparing with (40) shows that the noncommutativity has been shifted from the momenta to the coordinates.

As before, the lagrangian can be computed from an inverse Legendre transform. The result is,

$$
L=q_{i} \dot{x}_{i}+\frac{\theta}{2} \epsilon_{i j} q_{i} \dot{q}_{j}-\frac{k}{2} x_{i}^{2}-\frac{q_{i}^{2}}{2 m}
$$

leading to the set of equations (45). Eliminating the auxiliary variable $x_{i}$ yields,

$$
L=\frac{\dot{q}_{i}^{2}}{2 k}+\frac{\theta}{2} \epsilon_{i j} q_{i} \dot{q}_{j}-\frac{q_{i}^{2}}{2 m}
$$

from which equation (37) follows directly. 
The above analysis shows that noncommutativity is a manifestation of the presence of the magnetic field. In the extended set of coordinates this noncommutativity can occur either in $x_{i}$ or in $q_{i}$. However it is also possible to interpret $\left(x_{i}, q_{i}\right)$ as phase space variables. The common structure of the hamiltonian $\rho$ shows that $x_{i}$ and $q_{i}$ are coordinates and momenta, respectively. Interpreted this way, noncommutative spatial variables are just the dual of what happens for the momenta. Intrinsically there is no fundamental difference between them. Depending on the polarisation it is possible to have noncommuting momenta or coordinates. The dual nature is further realised by observing that while one polarisation yields the lagrangian in the $x$ variables, the other yields the lagrangian in the $q$ variables. The duality map $m \leftrightarrow k^{-1}$ connects the lagrangians (44) and (49); i.e., small ' $m$ ' in (44) corresponds to large ' $k$ ' in the latter. Now for small ' $m$ ', (44) reduces to a chiral oscillator for which $\left\{x_{i}, x_{j}\right\}=-B^{-1} \epsilon_{i j}$. In [6], a canonical derivation of this result is provided by observing that the hamiltonian (39) is now meaningful only if $q_{i}=0$. Imposing this as a constraint in (40), and then calculating the Dirac bracket, which, for any pair of variables $X_{i}, X_{j}$ subjected to the constraints $\Omega_{l}$, is defined as,

$$
\left\{X_{i}, X_{j}\right\}_{D B}=\left\{X_{i}, X_{j}\right\}-\sum\left\{X_{i}, \Omega_{l}\right\} W^{l m}\left\{\Omega_{m}, X_{j}\right\}
$$

where $W^{l m}$ is the inverse of the bracket among the constraints $\left(\left\{\Omega_{l}, \Omega_{m}\right\}\right)$,one finds [6],

$$
\left\{x_{i}, x_{j}\right\}_{D B}=-\sum\left\{x_{i}, q_{l}\right\} W^{l m}\left\{q_{m}, x_{j}\right\}=W^{i j}
$$

where $W^{i j}=-B^{-1} \epsilon_{i j}$. The noncommutative bracket is thus obtained as a Dirac bracket.

In the dual version small ' $m$ ' is replaced by large' $k$ '. Then (39) is meaningful for $x_{i}=0$. Imposing this as a constraint in (47), the Dirac bracket among $q_{i}$ is,

$$
\left\{q_{i}, q_{j}\right\}_{D B}=-\sum\left\{q_{i}, x_{l}\right\} \tilde{W}^{l m}\left\{x_{m}, q_{j}\right\}=\tilde{W}^{i j}
$$

where $\tilde{W}^{i j}=-\theta^{-1} \epsilon_{i j}$ is the inverse of $\left\{x_{i}, x_{j}\right\}$. This Dirac bracket is exactly the noncommutative bracket obtained direcly from (49) for large ' $k$ '. This completes the analysis of the dual structure.

We now show that the origin of noncommutativity is contained in a similar feature occurring in the chiral oscillators considered in the previous section. Two such oscillators $\left(L_{ \pm}\right)$rotating in clockwise and anticlockwise directions, respectively, with different frequencies, exactly simulate the Landau problem in the presence of the oscillator potential. This was shown in [10]. The magnetic field gets identified with the difference in the frequencies of the oscillators. Naturally, if the fequency of the oscillators is identical, the doublet of chiral oscillators just reduces to a two dimensional harmonic oscillator. Consider, therefore the equations of motion for the chiral $\operatorname{oscillators}\left(L_{ \pm}\right)$(see 21) with frequencies $\omega_{ \pm}$:

$$
\dot{z}_{i}=\omega_{+} \epsilon_{i j} z_{j}, \quad \dot{y}_{i}=-\omega_{-} \epsilon_{i j} y_{j}
$$

satifying the algebra,

$$
\left\{z_{i}, z_{j}\right\}=-\left\{y_{i}, y_{j}\right\}=\epsilon_{i j} ; \quad\left\{y_{i}, z_{j}\right\}=0
$$


Expressing the above set of equations in terms of a new set of variables,

$$
z_{i}+y_{i}=x_{i}
$$

we find

$$
\dot{x}_{i}=\epsilon_{i j}\left(\omega_{+} z_{j}-\omega_{-} y_{j}\right)
$$

To reproduce the first part of (36), put

$$
\epsilon_{i j}\left(\omega_{+} z_{j}-\omega_{-} y_{j}\right)=\frac{1}{m} q_{i}
$$

Solving for $y_{i}$ and $z_{i}$ in terms of $x_{i}$ and $q_{i}$ yields,

$$
\begin{aligned}
& y_{i}=\frac{1}{\omega_{+}+\omega_{-}}\left(\omega_{+} x_{i}+\frac{1}{m} \epsilon_{i j} q_{j}\right) \\
& z_{i}=\frac{1}{\omega_{+}+\omega_{-}}\left(\omega_{-} x_{i}-\frac{1}{m} \epsilon_{i j} q_{j}\right)
\end{aligned}
$$

If we take the time derivative of the difference of the above two equations and exploit (53)), the second equation of (36) is exactly reproduced with the following identification,

$$
k=m \omega_{+} \omega_{-}, \quad B=m\left(\omega_{+}-\omega_{-}\right)
$$

Expectedly the magnetic field is proportional to the difference of the frequencies.

Knowing the algebra of $z_{i}$ and $y_{i}$, it is easy to get the algebra of $x_{i}$ and $q_{i}$, which is given by,

$$
\begin{gathered}
\left\{x_{i}, x_{j}\right\}=0 \\
\left\{x_{i}, q_{j}\right\}=m\left(\omega_{+}+\omega_{-}\right) \delta_{i j} \\
\left\{q_{i}, q_{j}\right\}=m^{2}\left(\omega_{+}^{2}-\omega_{-}^{2}\right) \epsilon_{i j}=m\left(\omega_{+}+\omega_{-}\right) B \epsilon_{i j}
\end{gathered}
$$

where the mapping (60) has been used. Upto a trivial scaling this reproduces the algebra (40).

The hamiltonian of the composite system is obtained by adding the contributions from the two components and using (58, 59),

$$
H=H_{+}+H_{-}=\frac{\omega_{+}}{2} z_{i}^{2}+\frac{\omega_{-}}{2} y_{i}^{2}=\frac{1}{2 m\left(\omega_{+}+\omega_{-}\right)}\left(\frac{q_{i}^{2}}{m}+k x_{i}^{2}\right)
$$

which, modulo the scaling mentioned above, reproduces the desired expression (39).

Proceeding similarly it is possible to reproduce the polarisation (45) along with the associated algebra (匟). In this case define,

$$
z_{i}+y_{i}=q_{i}
$$

and,

$$
\epsilon_{i j}\left(\omega_{+} z_{j}-\omega_{-} y_{j}\right)=-k x_{i}
$$


so that the first equation in (45) is obtained. The same steps adopted earlier lead to the second equation in (45) with exactly the same normalization (60).

The algebra of $q_{i}$ and $x_{i}$ follows from (65) and (66):

$$
\left\{q_{i}, q_{j}\right\}=0 ; \quad\left\{x_{i}, q_{j}\right\}=\frac{\omega_{+}+\omega_{-}}{k} \delta_{i j} ; \quad\left\{x_{i}, x_{j}\right\}=\left(\frac{\omega_{+}+\omega_{-}}{k}\right) \theta \epsilon_{i j}
$$

and a simple scaling reproduces the desired algebra (47).

This section is concluded by providing a more general type of noncommutativity involving both $x_{i}$ and $q_{i}$. Consider the pair of first order equations,

$$
\begin{gathered}
\dot{x}_{i}=\alpha \epsilon_{i j} x_{j}+\beta q_{i} \\
\dot{q}_{i}=\lambda \epsilon_{i j} q_{j}+\gamma x_{i}
\end{gathered}
$$

which lead to the Landau type equations in both $x_{i}$ and $q_{i}$,

$$
\ddot{r}_{i}=(\alpha+\lambda) \epsilon_{i j} \dot{r}_{j}+(\alpha \lambda+\beta \gamma) r_{i} ; \quad r_{i}=x_{i}, q_{i}
$$

Following 't Hooft, a hamiltonian is constructed,

$$
H=\left(\alpha \epsilon_{i j} x_{j}+\beta q_{i}\right) \pi_{i}^{x}+\left(\lambda \epsilon_{i j} q_{j}+\gamma x_{i}\right) \pi_{i}^{q}
$$

where $\left(x_{i}, \pi_{i}^{x}\right)$ and $\left(q_{i}, \pi_{i}^{q}\right)$ are canonical pairs. The equations of motion $\dot{r}_{i}=\left\{r_{i}, H\right\}$ just yields (68, 69). As usual, this $H$ is not bounded from below. A positive definite $\rho$, commuting with $H$, has to be obtained. A natural choice is,

$$
\rho=\frac{1}{2}\left(a x_{i}^{2}+b q_{i}^{2}\right) ; \quad a, b \geq 0
$$

where $a \beta+b \gamma=0$ follows on demanding $\{\rho, H\}=0$. To satisfy the condition among the parameters $\beta$ is chosen to be negative $(\beta=-k)$. Then the desired hamiltonian becomes,

$$
H \rightarrow \rho=\frac{a}{2}\left(x_{i}^{2}+\frac{k}{\gamma} q_{i}^{2}\right)
$$

The linearity of the original pair of first order equations show that they remain unchanged under a scaling $r_{i} \rightarrow \sqrt{\frac{\gamma}{a}} r_{i}$. Moreover to make contact with the familiar form of the hamiltonian we set $\gamma=m^{-1}$ where $m$ is a mass parameter. Then the hamiltonian reduces to

$$
\rho=\frac{1}{2}\left(\frac{x_{i}^{2}}{m}+k q_{i}^{2}\right)
$$

It is easy to check that the corresponding algebra is,

$$
\left\{q_{i}, x_{j}\right\}=\delta_{i j} ; \quad\left\{x_{i}, x_{j}\right\}=\alpha \epsilon_{i j} ; \quad\left\{q_{i}, q_{j}\right\}=\frac{\lambda}{k} \epsilon_{i j}
$$


and the equations of motion $(68,69)$ reduce to,

$$
\begin{aligned}
& \dot{x}_{i}=\left\{x_{i}, \rho\right\}=\frac{\alpha}{m} \epsilon_{i j} x_{j}-k q_{i} \\
& \dot{q}_{i}=\left\{q_{i}, \rho\right\}=\lambda \epsilon_{i j} q_{j}+\frac{1}{m} x_{i}
\end{aligned}
$$

Both $x_{i}, q_{i}$ satisfy a noncommuting algebra.

The Lagrangian follows from an inverse Legendre transform. The matrix constructed from the brackets is,

$$
\Gamma_{i j}=\left[\left\{r_{i}, r_{j}\right\}\right]=\left(\begin{array}{cc}
\frac{\lambda}{k} \epsilon_{i j} & \delta_{i j} \\
-\delta_{i j} & \alpha \epsilon_{i j}
\end{array}\right)
$$

whose inverse is given by

$$
\Gamma^{i j}=\frac{1}{k-\lambda \alpha}\left(\begin{array}{cc}
k \alpha \epsilon_{i j} & -k \delta_{i j} \\
k \delta_{i j} & \lambda \epsilon_{i j}
\end{array}\right)
$$

Hence the lagrangian is given by,

$$
L=\frac{1}{2} r_{i} \Gamma^{i j} \dot{r}_{j}-\rho=\frac{1}{2(k-\lambda \alpha)}\left[\lambda \epsilon_{i j} x_{i} \dot{x}_{j}+\alpha k \epsilon_{i j} q_{i} \dot{q}_{j}+2 k x_{i} \dot{q}_{i}\right]-\frac{1}{2}\left[\frac{x_{i}^{2}}{m}+k q_{i}^{2}\right]
$$

Obviously $\frac{\lambda \alpha}{k}=1$ is a special point manifesting a degeneracy. This particular point has been discussed in details elsewhere [11]. The equations of motion obtained from this lagrangian are compatible with (76, 77).

The rotational symmetry of the hamiltonian $\rho$ suggests that the angular momentum operator $J$ has the conventional algebra with $x_{i}$ and $q_{i}$,

$$
\left\{J, r_{i}\right\}=\epsilon_{i j} r_{j} ; \quad r_{i}=x_{i}, q_{i}
$$

The structure of $J$ which yields these equations, from the basic brackets (75), is given by,

$$
J=\frac{1}{2(k-\lambda \alpha)}\left(\lambda x_{i}^{2}+\alpha k q_{i}^{2}+2 k \epsilon_{i j} q_{i} x_{j}\right)
$$

Actually this expression for $J$ follows from (80) by following a standard canonical prescription,

$$
J=\epsilon_{i j}\left(x_{i} \pi_{j}^{x}+q_{i} \pi_{j}^{q}\right)
$$

where,

$$
\begin{gathered}
\pi_{i}^{x}=\frac{\partial L}{\partial \dot{x}_{i}}=-\frac{\lambda}{2(k-\lambda \alpha)} \epsilon_{i j} x_{j} \\
\pi_{i}^{q}=\frac{\partial L}{\partial \dot{q}_{i}}=\frac{k}{2(k-\lambda \alpha)}\left(2 x_{i}-\alpha \epsilon_{i j} q_{j}\right)
\end{gathered}
$$

are the respective conjugate momenta. 


\section{Noncommutative field theory in the lowest Landau level}

In a recent paper [6] noncommutativity in a magnetohydrodynamical model was studied by taking the vanishing limit of a mass parameter, in analogy with the lowest Landau level problem. A similar study will be done here, based on the ideas presented earlier.

A field theory is obtained by introducing electron creation and annihilation operators in terms of which the density operator is $\eta(x)=\psi^{\dagger} \psi(x)$. Now the hamiltonian $h$ of a particle in the lowest Landau level is just the projection of potential to that level [12],

$$
h=\int d^{2} x V(x) \eta(x)
$$

since the kinetic term, after projection, gets quenched.

If a particle substructure is given to a fluid, then $\eta(x)$ is the density of the fluid satisfying the continuity equation,

$$
\dot{\eta}=-\partial_{i}\left(\eta v_{i}\right)
$$

The hamiltonian leading to this equation is,

$$
H=-\int d^{2} x \partial_{i}\left(\eta v_{i}\right) \pi^{\eta}
$$

where $\left(\eta, \pi^{\eta}\right)$ is a canonical pair. As usual, this is not bounded from below. The physical hamiltonian would be the operator commuting with $H$. In our case this is given by (86) so that $\{h, H\}=0$ yields,

$$
\int d^{2} x V(x) \partial_{i}\left(\eta v_{i}\right)(x)=0
$$

Upto a normalization $N$, a solution for $v_{i}(x)$ is,

$$
v_{i}(x)=N \epsilon_{i j} \partial_{j} V(x)
$$

With this $v_{i}(x)$, the continuity equation (87) is reproduced by bracketing with $h(\dot{\eta}=\{\eta, h\})$ provided,

$$
\{\eta(x), \eta(y)\}=-N \epsilon_{i j} \partial_{i} \eta \partial_{j} \delta(x-y)
$$

revealing a noncommutativity among the density operators. Exactly the same structure follows for the Landau problem if the explicit form for $\eta(x)$ and the noncommuting brackets among $x$ is used, with the identification,

$$
N=\frac{c}{e B}
$$

The bracket (91) with the normalisation (92) was obtained in [6] by using the continuity equation and Euler equation in the massless limit. 


\section{Concluding remarks}

Let us now digress on the significance of our findings. The Landau model lagrangians with an oscillator potential, expressed in their usual second order forms (44) or (49) obviously satisfy a conventional commutative algebra. In the standard treatments [2] one introduces a noncommutative plane by hand and subsequently discusses the model on that plane. Here we show that, by transforming the second order system to a first order one, by introducing an additional variable, the noncomutative plane is naturally induced. Indeed, by following 't Hooft's analysis done in a different context, we have provided a systematic way of obtaining the various noncommutative strutures on the plane. In this connection we might recall that a "natural explanation" for the occurrence of noncommutativity in the Landau problem is attributed to the presence of the magnetic field. However, this effect is only found for the lowest Landau level in which case the original second order system is effectively replaced by a first order one. This is the origin of the change in the symplectic structure. Something similar happens here where a passage from the second order to the first order system has been effected. Our conclusions are further confirmed by the fact that all the noncommuting structures were also obtained as a superposition of two chiral oscillators, which are essentially first order systems, moving in opposite (clockwise and anticlockwise) directions. Since the difference in the frequencies of the two rotations is actually proportional to the magnetic field, the connection of this approach with the one where noncommutaivity is just introduced through the presence of a magnetic field gets established.

A consequence of our analysis has been that the manifestation of the noncommutativity in the coordinates or in the momenta is shown as a dual aspect of the same phenomenon. Indeed we established a direct connection between the noncommutativity parameter $B$ (the magnetic field) in the momentum algebra with the corresponding parameter $\theta$ in the coordinate algebra

through the relation $\theta=\frac{B}{k m}$. This is again reminiscent of an analogous connection found in the discussions of noncommutativity in the context of open string quantisation [1]. Furthermore, the critical point (where the density of states becomes infinte) found in the literature [11] in the analysis comprising noncommutativity in both coordinates and momenta, was also obtained here. At this point it was shown that the symplectic matrix does not have an inverse so that the transition from the hamiltonian to the lagrangian by an inverse Legendre transform was not feasible.

We conclude this paper by briefly suggesting the restrictions on the Landau orbits brought about by the imposition of the constraints that eventually led to a bounded hamiltonian from the unbounded one. Thus if the period of the cyclotron orbit in a chiral oscillator be a function $T(\rho)$ of $\rho$, where the constraint leads to the physical hamiltonian $\rho=H$, then [3]

$$
e^{-i H T}|\psi>=| \psi>
$$

so that,

$$
\rho T(\rho)=2 \pi n, \quad n \in \mathcal{Z}
$$

This means that the orbits in the Landau problem with an oscillator potential comprise two 
pieces (see 53); clockwise orbits satisfying the periodicity condition,

$$
\frac{\omega_{+}}{2} z_{i}^{2} T(z)=2 \pi n, \quad n \in \mathcal{Z}
$$

and anticlockwise orbits obeying,

$$
\frac{\omega_{-}}{2} y_{i}^{2} T(y)=2 \pi m, \quad m \in \mathcal{Z}
$$

As a final remark we mention some recent works [13] which reveal the continuing interest in quantum mechanics on the noncommuting plane.

\section{Acknowledgement}

I thank Gerard 't Hooft for a useful correspondence.

\section{References}

[1] For recent reviews, see, M.R. Douglas and N.A. Nekrasov, hep-th/0106048; R.J. Szabo, hep-th/0109162.

[2] J. Lukierski, P. Stichel and W. Zakrzewski, Ann. Phys. 260 (1997) 224; V. P. Nair and A. P. Polychronakos, Phys. Lett. B505 (2001) 267; A. Hatzinikitas and I. Smyrnakis, hep-th/0103074; J. Gamboa, M. Loewe and J. Rojas, hep-th/0010220.

[3] G. 't Hooft, Class. Quant. Grav.16(1999)3263; hep-th/0003005; hep-th/0105105.

[4] M. Blasone, P. Jizba and G. Vitiello, hep-th/0007138.

[5] M. Eliashvili and G. Tsitsishvili, Intl. J. Mod. Phys. B14 (2000) 1429; L. Susskind, hepth/0101029; A. P. Polychronakos JHEP 0104 (2001) 011, hep-th/0103013; S. Hellerman and M. Van Raamsdonk, hep-th/0103179.

[6] Z. Guralnik, R. Jackiw, S. Y. Pi and A. P. Polychronakos, hep-th/0106044.

[7] For a recent review, see, S. Girvin, cond-mat/9907002.

[8] R. Banerjee and S. Ghosh, J. Phys. A31 (1998) L603.

[9] S. Deser and R. Jackiw, Phys. Lett. B139 (1984) 371; R. Banerjee and H.J. Rothe, Nucl. Phys. B447 (1995) 183.

[10] R. Banerjee and S. Kumar, Phys. Rev. D60 (1999) 085005.

[11] See, for example, the second and third papers in ref.3.

[12] V. Gurarie and A. Zee, cond-mat/0008163.

[13] C. Duval and P. A. Horvathy, hep-th/0106089; C. D. Fosco and A. Lopez, hep-th/0106136 